\title{
ASENKRON MOTORUN ALAN ZAYIFLAMA BÖLGESINDE KAYAN KİP DENETÇİ TABANLI HIZ-ALGILAYICISIZ DOĞRUDAN VEKTÖR KONTROLÜ
}

\author{
Remzi İNAN ${ }^{1}$ (ORCID: 0000-0003-1717-3875)* \\ ${ }^{I}$ Niğde Ömer Halisdemir Üniversitesi, Mühendislik Fakültesi, Elektrik-Elektronik Mühendisliği Bölümü
}

Geliş / Received: 21.01 .2019 Kabul / Accepted: 04.07.2019

\begin{abstract}
ÖZ
Bu çalışmada, asenkron motorların (ASM'lerin) hız-algılayıcısız kayan kip denetçi (KKD)-tabanlı doğrudan vektör kontrolünde (DVK'sında) rotor akısının $\alpha \beta$ - stator duran eksen takımı bileşeni $\left(\varphi_{r \alpha}\right.$ ve $\left.\varphi_{r \beta}\right)$, rotor mekanik açısal hızı $\left(\omega_{m}\right)$, yük momenti $\left(t_{L}\right)$, mıknatıslama endüktansı $\left(L_{m}\right)$ ve rotor direnci $\left(R_{r}^{\prime}\right)$ kestirimi için indirgenmiş dereceli genişletilmiş Kalman filtresi (IDGKF) temelli yeni bir kestirici önerilmiştir. Önerilen IDGKF kestiricisinin kestirim başarımı ve bu kestiricinin hız-algılayıcısız KKD-tabanlı DVK'da kullanılması ile de sürücü sisteminin kontrol başarımı benzetim ortamında test edilmiştir. Benzetim ortamında gerçekleş̧irilen kestirim ve kontrol başarımına ilişkin testlerde, ASM sıfır hızdan anma hızına $\left(n_{b}\right)$ ve anma hızının üzerindeki alan zayıflama bölgesini de kapsayan geniş bir hız aralığında çalıştııılmış ve tüm hız aralıklarında zorlayıcı durum ve parametre değişimleri meydana getirilmiştir. Bu zorlayıcı referans değişimleri altında, benzetim ortamından elde edilen kestirim sonuçları, önerilen IDGKF algoritmasının yüksek bir kestirim başarımına ve dolayısıyla ASM'nin KKD-tabanlı DVK'sının sıfır hızdan alan zayıflama bölgesini de içeren geniş bir hız aralığında yüksek başarımlı bir kontrol performansına sahip olduğunu göstermektedir. Böylelikle, ilk kez bu çalı̧̧mada önerilen İDGKF temelli yeni kestiricinin, ASM'nin hız-algılayıcısız sürücü sisteminin durum ve parametre değişimlerine karşı daha gürbüz bir yapı sergilemesine olanak sağladığı gösterilmiştir.
\end{abstract}

Anahtar kelimeler: ASM, alan zayıflama bölgesi, IDGKF, KKD

\section{SPEED-SENSORLESS SLIDING MODE CONTROLLER BASED DIRECT VECTOR CONTROL OF INDUCTION MOTOR IN FIELD WEAKENING REGION}

\begin{abstract}
In this paper, a new reduced order extended Kalman filter (ROEKF)- based estimator is proposed for the estimations of $\alpha \beta$ - stator stationary axis components of rotor flux $\left(\varphi_{r \alpha}\right.$ ve $\left.\varphi_{r \beta}\right)$, rotor mechanical angular velocity $\left(\omega_{m}\right)$, load torque $\left(t_{L}\right)$, magnetizing inductance $\left(L_{m}\right)$, and rotor resistance $\left(R_{r}^{\prime}\right)$ which is used in speedsensorless sliding mode controller (SMC)-based direct vector control (DVC) of induction motors (IMs). The estimation performance of the proposed ROEKF estimator and the control performance of the drive system are also tested in simulation by using this estimator in the speed-sensorles SMC-based DVC. In the simulation test on the estimation and control performance of whole drive system, the IM is operated from zero speed to rated speed $\left(n_{b}\right)$ and above the $n_{b}$ which is known as field weakening region in the literature, and state/parameter changes are made at all operation region. Under these coercive changes of the states and parameters, the simulation results show that the proposed ROEKF estimator and SMC-based DVC of IM have high estimation and control performance in a wide speed range including zero speed to field weakening region. In this way, it is shown that the ROEKF-based new estimator which is proposed for the first time in this study, allows the speedsensorless drive system of IM to exhibit a more robust structure against the state and parameter changes.
\end{abstract}

Keywords: IM, field-weakening region, ROEKF, SMC

*Corresponding author / Sorumlu yazar. Tel.: 038822527 99; e-mail / e-posta: rinan@ohu.edu.tr 


\section{GİRIŞ}

ASM'ler, düşük bakım maliyetleri, dayanıklı olmaları, atalet momentlerinin düşük olması, yüksek moment indükleme kapasitesine ve düşük moment salınımlarına sahip olmaları nedeni ile elektrikli araçlarda, vinç, robotik ve endüstriyel uygulama alanlarında yaygın olarak kullanılmaktadır. Doğrudan akı yönlendirmeli kontrol (AYK) ya da DVK, yüksek bir hız aralığında yüksek başarımlı bir kontrol performansına sahip olması nedeni ile ASM'lerin dinamik kontrolünde literatürde en çok tercih edilen kontrol yöntemidir [1]. DVK ya da AYK yöntemi doğru akım motorlarında olduğu gibi ASM'nin moment ve akı kontrolünün birbirinden bağımsız olarak gerçekleştirilebilmesine olanak sağlamaktadır.

PID denetçi yapıları, basit yapıları ve yüksek kontrol performansları nedeni ile DVK yönteminde alan yönlendirme koşulunun sağlanması amacı ile kullanılmaktadır [2]. Öte yandan, PID denetçi yapısının parametrelerinin ve katsayılarının belirlenmesinde, öngörülemeyen parametre değişiklikleri, harici yük bozulmaları ve modellenmemiş doğrusal olmayan dinamikler nedeni ile oluşan zorluklar [3, 4] araştırmacıları farklı alternatif çözümler aramaya yönlendirmektedir. Bu nedenle, ASM'nin DVK'sının bu zorluklara karşı kontrol performansını artırmak için literatürde, uyarlamalı giriş/çıkış doğrusallaştırma kontrolü [5], öngörü tabanlı kontrol [6, 7], uyarlamalı kontrol [8-10], gürbüz kontrol [11], bulanık kontrol [12], yapay sinir ağları [13, 14] ve KKD [4, 15-18] gibi birçok yaklaşım önerilmiştir.

Bozuculardan etkilenmemesi, gürbüz olması ve tasarım kriterinin sağladığı uygulama kolaylığı gibi özellikleri ile KKD'ler oldukça yüksek bir kontrol başarımına sahiptirler [3, 19, 20, 21, 22]. Ayrıca KKD, belli bir örnekleme frekansı ile sayısal işlemciler üzerinde ayrık zamanlı olarak gerçekleştirilen kontrol sistem tasarımında sistem durum yörüngesini durum uzayındaki kayan yüzeye sürmek için süreksiz kontrol yasalarını kullanmaktadır [3, 4, 22, 23, 24]. Bununla birlikte, kontrol sistemlerinin yüksek örnekleme frekans1 ile gerçekleştirilmesi ve kontrol sisteminin hızlı dinamik değişimlerinin ihmal edilerek göz ardı edilmesi nedeni ile KKD’lerde çatırdama (chattering) probleminin ortaya çıktığ 1 bilinmektedir [3, 23, 25, 26]. Literatürde ASM'lerin KKD'sindeki çatırdama problemini çözmek ve ayrıca bu kontrol yönteminin başarımını artırmak için çeşitli çalışmalar yapılmıştır ve bu çalışmalar [3, 4, 23, 24, 26]'da özetlenmiştir. Bu çalışmalar içerisinde, [3]'te çatırdama probleminin azaltılmasına yönelik önerilmiş olan KKD, kararlı ve gürbüz bir yapıya sahip olmasıyla birlikte mikrodenetleyici tabanlı sayısal platformlarda kolay uygulanabilir olması nedeni ile ASM'lerin KKDtabanlı kontrolüne yönelik olarak önerilmiş olan çalışmalar arasında en etkin kullanım alanına sahip olandır.

ASM'lerin çalışma bölgeleri, sıfır hızdan anma hızına $\left(n_{b}\right)$ kadar olan çalışma bölgesi ve anma hızının üzerindeki alan zayıflama bölgesi olmak üzere ikiye ayrılmıştır [27]. Sıfır hız ile anma hızı arasındaki ilk çalışma bölgesinde maksimum moment sabit tutulmakta olup, ASM'nin anma gücü stator frekansı ile ilişkilidir. Fakat anma hızının üzerindeki alan zayıflama bölgesinde doyum etkisi nedeni ile ASM'nin anma gücü sabit tutularak maksimum moment azaltılmalıdır.

Anma hızının altındaki çalışma bölgesinde, ASM'nin akısı ve $L_{m}$ sabit tutularak ASM'nin AYK'sı sağlanmaktadır. Alan zayıflama bölgesinde ise AYK'nın sağlanabilmesi için motor akısı, ASM'nin rotor mekanik hızı ile ters orantılı olarak azaltılmalıdır $\left(1 / \omega_{r}\right)$. ASM'nin akı bilgisindeki değişim ASM'nin doyum derecesini yansıtmakta olup bu sebeple de $L_{m}$ değişmektedir [28, 29]. Ayrıca, [30, 31]'de belirtildiği gibi, ASM'nin manyetik akı yoğunluğu-manyetik alan şiddeti (B-H) eğrisinde motor akı referansının azaltılması ile çalışma bölgesi orijine yaklaşmakta olup $L_{m}$ değeri artmaktadır.

ASM'lerin alan yönlendirmeli sürücü sistemlerinde, tüm çalışma bölgelerinde ASM'nin algılaycısız kontrolünün sağlanması bazı problemler içermektedir. ASM'nin hız-algılayıcız kontrolünde rotor mekanik hız bilgisinin ve akı bilgisinin kesin değerlerine ihtiyaç duyulmakta olup, bu değerler gözlemleyici ya da kestirici yapıları kullanılarak elde edilmeye çalışılmaktadır. Ayrıca, gözlemleyici ve kestirici yapılarının tasarımında da bu yapıların durum ve parametre değişimlerine karşı kararlı ve gürbüz bir yapı sergilemeleri sağlanmalıdır. Özellikle alan zayıflama bölgesinde çalışma esnasında, rotor sıcaklığına bağlı olarak $R_{r}^{\prime}$ 'de meydana gelen değişimler ve yüksek stator frekansı, ASM'nin alan yönlendirme koşulunun gerçekleştirilmesini bir hayli zorlaştırmaktadır. $\mathrm{Bu}$ nedenle, alan zayıflatma işlemi sırasında, ASM'nin rotor zaman sabitinin $\left(T_{r}\right)$ doğru şekilde bilinmesi gerekliliği nedeni ile $L_{m}$ ve $R_{r}^{\prime}$ eş-zamanlı olarak kestirilmelidir. Ayrıca, kestirici tabanlı hızalgılayıcısız sürücü sistemlerinde, eğer $\omega_{m}$ bir durum olarak kestirilirse, ASM'nin $t_{L}$ değeri de kestirilebilmektedir. Bu durumlara ve parametrelere ilaveten, stator sarg1 sıcaklığına bağlı stator direncindeki $\left(R_{S}\right)$ değişiklikler, gerilim düşümü etkisinin düşük veya sıfır hızda çalışma sırasında önem arz etmesi, özellikle düşük ve sıfır hızda meydana gelebilecek $R_{s}$ değişimlerinin tahmin edilmesini gerektirmektedir. Ancak, [32, 33]'te belirtildiği gibi, ASM'nin alan zayıflama bölgesinde çalışması sırasında $L_{m}$ ve $R_{r}^{\prime}$ 'nin kestirimi, $R_{s}$ 'nin kestiriminden daha önemlidir, çünkü $R_{s}$ 'den dolayı oluşan gerilim düşümü etkisi yüksek hızlarda azalmaktadır.

Literatürde, alan zayıflama bölgesinde ASM'nin durum ve parametre kestirimlerini içeren çeşitli çalışmalar bulunmaktadır [27, 32-47]. Bu çalışmalardan bazıları, ASM'nin hız-algılayıcısız AYK'sı için önerilmiş olup [27, $32,34-42]$, diğer bir grup çalışmada ise durum ve parametre kestiriminde hız algılayıcısı kullanılmaktadır [33, 
43-47]. [27] 'de, ASM'lerin sıfır hızdan anma hızına kadar geniş bir hız aralığında ve aynı zamanda alan zayıflama bölgesinde hız-algılayıcısız DVK'sı için yeni bir çift girişli-GKF (ÇG-GKF) tabanlı kestirim yöntemi önerilmiştir. Önerilen ÇG-GKF ile $i_{s \alpha}, i_{s \beta}, \varphi_{r \alpha}, \varphi_{r \beta}, \omega_{m}, t_{L}, R_{s}, R_{r}^{\prime}$ ve $L_{m}$ kestirimleri eş-zamanlı olarak gerçekleştirilmiştir. Kestirilen durum ve parametreler gerçek ya da referans değerlerine başarılı bir şekilde yakınsamaktadır. [32]'de, rotor akısının $d q$ - döner eksen takımı bileşenleri ( $\varphi_{r d}$ ve $\left.\varphi_{r q}\right)$ rotor akısının türetilmesi yöntemi ile, $\omega_{m}$ baskın rotor oluk harmonikleri yöntemi ile ve $T_{r}$ ise ayarlanmış parçacık sürü optimizasyonu ile kestirilmiştir. Fakat, alan zayıflama bölgesinde herhangi bir kestirim sonucu verilmemiştir. Stator akımının $d q$ bileşenleri $\left(i_{s d}\right.$ ve $\left.i_{s q}\right), \varphi_{r d}, \varphi_{r q}$ ve $\omega_{m}$ değiş̧imleri ayrıklaştırılmış hız-uyarlamalı tam dereceli gözlemleyici ile kestirilmiştir [34, 35]. Fakat, $R_{r}^{\prime}$ ve $L_{m}$ değişimleri dikkate alınmamıştır. [36, 37]'de $i_{s \alpha}, i_{s \beta}, \varphi_{r \alpha}, \varphi_{r \beta}, \omega_{m}$ ve $t_{L}$ değişimleri GKF-tabanlı bir kestirim algoritması ile kestirilmiş olup, geçici-hallerdeki hız kestirim başarımı oldukça düşüktür. ASM'nin hız bilgisi zit elektromotor kuvvet tabanlı model uyarlamalı sistem (MUS) ile [38]'de ve gerilim tabanlı MUS yapısı ile de [39]'da kestirilmiştir. $\omega_{m}$ 'ye ait gürültülü kestirim sonuçları ASM'nin dinamik kontrolünü olumsuz yönde etkilemektedir. [40, 41]'de, $L_{m}$ değişimleri hem kontrol sistemine hem de $i_{s \alpha}, i_{s \beta}, \varphi_{r \alpha}, \varphi_{r \beta}$ ve $\omega_{m}$ kestirimi için önerilen kayma kipli gözlemleyiciye (KKG'ye) güncellenmiştir. $L_{m}$ 'de meydana gelen değişimler ASM'nin birime indirgenmiş mıknatıslama akısı ve iki farklı bağımsız katsayının uygun seçimi ile gerçekleştirilen bir analitik fonksiyon yardımı ile oluşturulmuş ASM'nin ters mıknatıslama eğrisi kullanılmıştır. Yalnızca [40]'da $R_{S}$ değişimi bir KKD ile kestirilmiş olup çok kısa bir zaman aralığını kapsayan bir sonuç sunulmuştur. [42]'de ASM'nin hız-algılayıcısız DVK'sı için önerilen GKF ile $i_{s \alpha}$, $i_{s \beta}, \varphi_{r \alpha}, \varphi_{r \beta}, \omega_{m}, R_{r}^{\prime}$ ve $L_{m}$ kestirimi gerçekleştirilmiş olup $\omega_{m}$ sabit parametre olarak kestirildiği için $t_{L}$ kestirimi mümkün olmamaktadır.

Literatürde, hız-algılayıcısı kullanılarak önerilen kestirim yöntemleri içerisinde, [33]'te MRAS yapısı ile motorun bilinen mıknatıslama eğrisi kullanılarak ASM'ye ait $T_{r}$ kestirimi gerçekleştirilmiştir. Ancak bu çalışmada ASM'nin alan zayıflama bölgesinde çalışması incelenmemiştir. [43]'te, $i_{s \alpha}, i_{s \beta}, \varphi_{r \alpha}, \varphi_{r \beta}, R_{s}$ ve $R_{r}^{\prime}$ kestirimi için GKF-tabanlı bir kestirim yöntemi ile birlikte, çevrimiçi $L_{m}$ kestirimi için ASM'nin ters mıknatıslama eğrisinin analitik fonksiyonu kullanılarak bir karma kestirim yöntemi önerilmiştir. $i_{s d}, i_{s q}, \varphi_{r d}$ ve $\varphi_{r q}$ kestiriminin doğrusal olmayan bir kestirici ile ve $L_{m}$ kestiriminin ASM'nin ters mıknatıslama eğrisinin analitik fonksiyonu kullanılarak gerçekleştirildiği çalışma [44]'de sunulmuştur. [45]'de, ASM model denklemlerinden türetilen yeni bir $L_{m}$ hesaplama yöntemi, rotor akı sapma yöntemi ile birlikte önerilmiştir. [46] 'da, kendi kendine devreye alma sırasında işaret zerketme yöntemi kullanılarak $L_{m}$ 'nin çevrimdışı tanımlaması elde edilmiştir. [47] 'deki yazarlar, giriş vektörünün $\varphi_{r \alpha}, \varphi_{r \beta}, R_{r}^{\prime}$ ve $L_{m}$ tahminleri için yeni bir ASM modelinden türetilen, indirgenmiş dereceli GKF (IDGKF) algoritması önermektedir. Önerilen IDGKF algoritması, geniş bir hız aralığında ASM'nin DVK'sı üzerinde benzetim ortamında test edilmiş̧ir ve benzetim sonuçları, IDGKF'nin kestirim performansını kanıtlamaktadır.

GKF, diğer yöntemlerin aksine durum ve parametre tahmini için stokastik tabanlı bir yaklaşım kullanır. GKF, hesaplama yüküne rağmen, ölçüm ve sistem gürültülerini dikkate alarak durumların ve parametrelerin eşzamanlı olarak kestirilmesine olanak sağlar [48]. ASM'nin hı-algılayıcısız kontrolünde kullanılan GKF algoritmaları tam dereceli GKF ve İDGKF olarak iki gruba ayrılmaktadır. Yüksek kestirim başarımına sahip İDGKF yöntemi, tam dereceli GKF yönteminin giriş vektörünün doğrusallaştırılması gerekliliği ve matrisel işlem karmaşasından dolayı içerdiği hesap yükünün azaltılması amacı ile önerilmiştir [49].

Bu çalışmanın temel katkısı, yeni bir çevrimiçi durum ve parametre kestiricisi ile ASM'nin hız-algılayıcısız KKD-tabanlı DVK'sının alan zayıflama bölgesinde kontrol performansını arttırmaktır. Bu amaçla, ASM'nin $\varphi_{r \alpha}, \varphi_{r \beta}, \omega_{m}, t_{L}, L_{m}$ ve $R_{r}^{\prime}$ durum ve parametrelerinin kestirimi için yeni bir IDGKF yöntemi önerilmiştir. ASM durum ve parametrelerinde meydana getirilen basamak şeklindeki ya da doğrusal şekildeki referans değişimler ile ASM'nin hız-algılayıcısız KKD-tabanlı DVK'sının ve yeni önerilen İDGKF yönteminin kontrol ve kestirim başarımları Matlab/Simulink benzetim ortamında test edilmiştir. Benzetim sonuçları, önerilen İDGKF'nin hem sıfir hızda hem de alan zayıflama bölgesinde yüksek kestirim başarımına sahip olduğunu ve bu nedenle ASM'nin KKD-tabanlı DVK'sının geniş bir hız aralığında gürbüz bir şekilde gerçekleştirilebileceğini göstermektedir.

\section{ROTOR AKISI TABANLI ASM MODELLERI}

Bu çalışmada, $\varphi_{r \alpha}, \varphi_{r \beta}, \omega_{m}, t_{L}, L_{m}$, and $R_{r}^{\prime}$ durum ve parametrelerinin kestirildiği İGKF algoritmasının geliştirilmesi için ASM'nin $\alpha \beta$ - stator duran eksen takımındaki rotor akısı tabanlı ASM modeli kullanılmıştır. ASM'nin genelleştirilmiş modeli (1) ve (2)'deki gibi verilmiştir: 


$$
\begin{aligned}
\dot{\mathbf{x}}_{\mathrm{g}} & =\mathbf{f}_{\mathrm{g}}\left(\mathbf{x}_{\mathrm{g}}, \mathbf{u}_{\mathrm{g}}\right)+\mathbf{w}_{\mathrm{g}} \\
& =\mathbf{A}_{\mathrm{g}}\left(\mathbf{x}_{\mathrm{g}}\right) \mathbf{x}_{\mathrm{g}}+\mathbf{B}_{\mathrm{g}} \mathbf{u}_{\mathrm{g}}+\mathbf{w}_{\mathrm{g}} \\
\mathbf{Z}_{\mathrm{g}} & \left.=\mathbf{h}_{\mathrm{g}}\left(\mathbf{x}_{\mathrm{g}}\right)+\mathbf{v}_{\mathrm{g}} \text { (Ölçüm Eşitliği }\right) \\
& =\mathbf{H}_{\mathrm{g}} \mathbf{x}_{\mathrm{g}}+\mathbf{v}_{\mathrm{g}}
\end{aligned}
$$

Burada, $\mathbf{x}_{\mathrm{g}}$, geniş̧letilmiş durum vektörüdür. $\mathbf{f}_{\mathrm{g}}$, durum ve girişlerin doğrusal olmayan fonksiyonudur. $\mathbf{A}_{\mathrm{g}}$, sistem matrisidir. $\mathbf{B}_{\mathrm{g}}$, giriş matrisidir. $\mathbf{u}_{\mathrm{g}}$, kontrol giriş vektörüdür. $\mathbf{w}_{\mathrm{g}}$, sistem gürültüsüdür. $\mathbf{Z}_{\mathrm{g}}$, ölçüm vektörüdür. $\mathbf{h}_{\mathrm{g}}$, çıkışların fonksiyonudur. $\mathbf{H}_{\mathrm{g}}$, ölçme matrisidir. $\mathbf{v}_{\mathrm{g}}$, ölçme gürültüsüdür. (1) ve (2)'de genelleştirilmiş modeli verilen rotor akısı tabanlı ASM modelinin ayrıntılı durum uzay gösterimi (5) ve (6)'da verilmiştir.

Ölçülemeyen durum ve parametrelerin kestiriminin gerçekleştirildiği İDGKF algoritmasının tasarımında kullanılan indirgenmiş ASM modelinin genelleştirilmiş ifadesi (3) ve (4)'te sunulmuştur.

$$
\begin{aligned}
\dot{\mathbf{x}}_{\mathrm{i}} & =\mathbf{f}_{\mathrm{i}}\left(\mathbf{x}_{\mathrm{i}}, \mathbf{u}_{\mathrm{i}}\right)+\mathbf{w}_{\mathrm{i}} \\
& =\mathbf{A}_{\mathrm{i}}\left(\mathbf{x}_{\mathrm{i}}\right) \mathbf{x}_{\mathrm{i}}+\mathbf{B}_{\mathrm{i}} \mathbf{u}_{\mathrm{i}}+\mathbf{w}_{\mathrm{i}} \\
\mathbf{Z}_{\mathrm{i}} & =\mathbf{h}_{\mathrm{i}}\left(\mathbf{x}_{\mathrm{i}}\right)+\mathbf{v}_{\mathbf{i}} \text { (Ölçüm Eşitliği) } \\
& =\mathbf{H}_{\mathrm{i}} \mathbf{x}_{\mathrm{i}}+\mathbf{v}_{\mathrm{i}}
\end{aligned}
$$

Burada (.) $)_{\mathrm{i}}$, indirgenmiş modele ait vektör ve matrisleri belirtmektedir. (3) ve (4)'te genelleştirilmiş modeli verilen rotor akısı tabanlı ASM modelinin ayrıntılı durum uzay gösterimi (7) ve (8)'de verilmiştir.

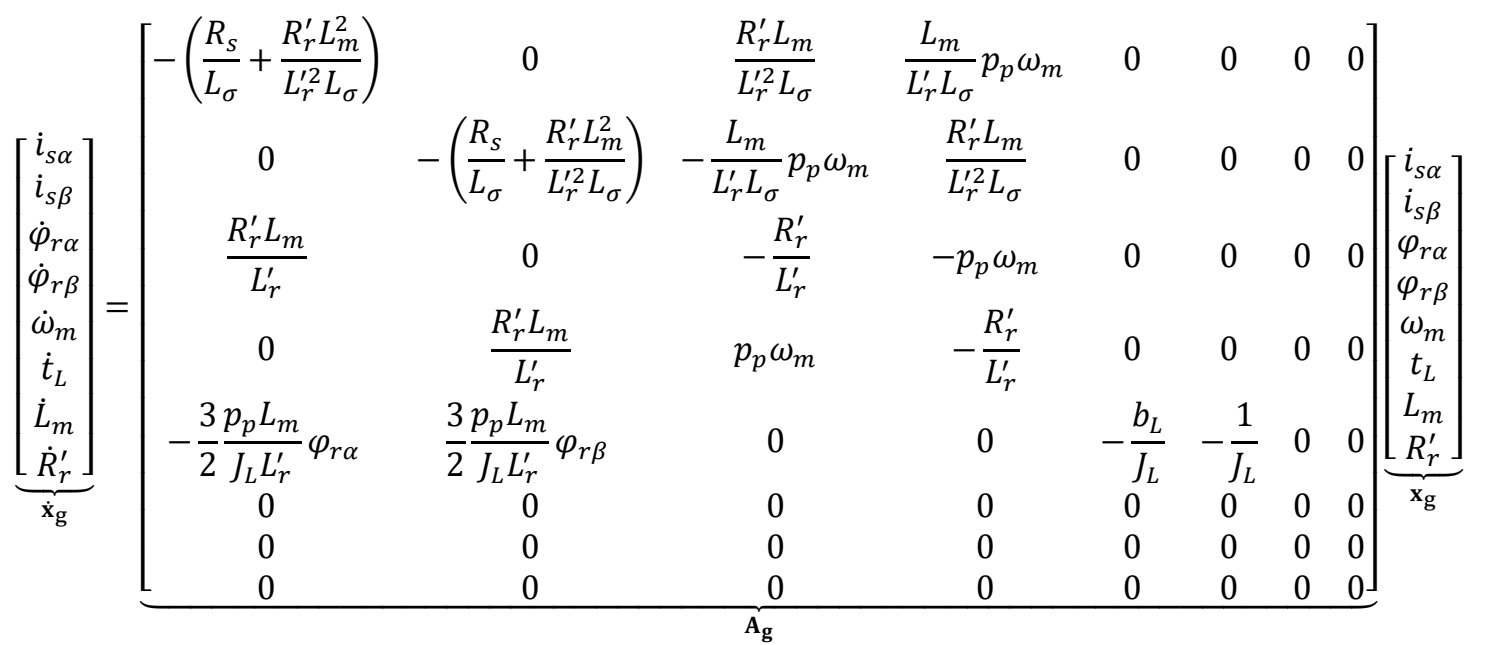

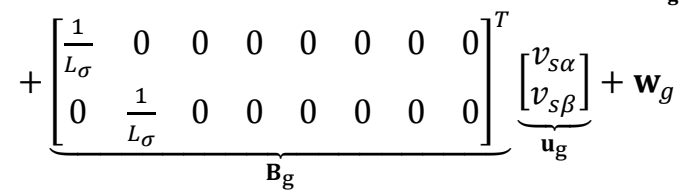

$$
\begin{aligned}
& \underbrace{\left[\begin{array}{c}
i_{s \alpha} \\
i_{s \beta}
\end{array}\right]}_{\mathbf{Z}_{\mathbf{i}}}=\underbrace{\left[\begin{array}{llllllll}
1 & 0 & 0 & 0 & 0 & 0 & 0 & 0 \\
0 & 1 & 0 & 0 & 0 & 0 & 0 & 0
\end{array}\right]}_{\mathbf{H}_{\mathbf{g}}} \underbrace{\left[\begin{array}{c}
i_{s \alpha} \\
i_{s \beta} \\
\varphi_{r \alpha} \\
\varphi_{r \beta} \\
\omega_{m} \\
t_{L} \\
L_{m} \\
R_{r}^{\prime}
\end{array}\right]}_{\mathbf{x}_{\mathbf{g}}}+\mathbf{v}_{\mathbf{g}}
\end{aligned}
$$




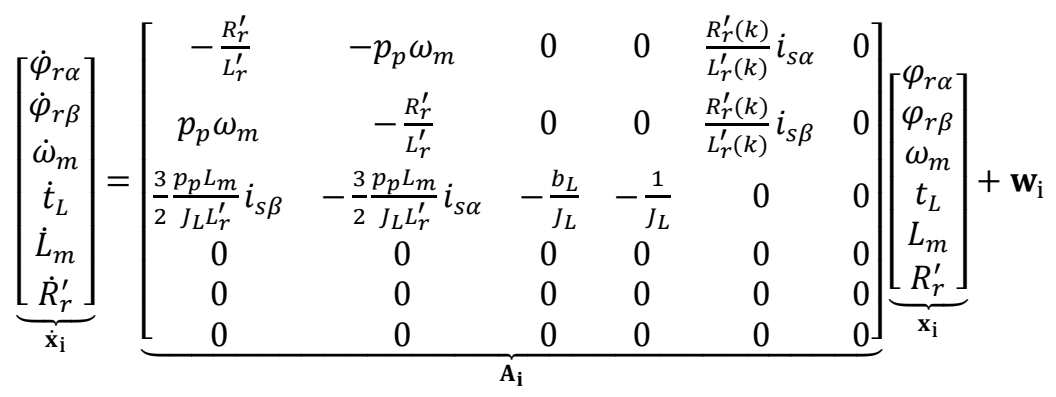

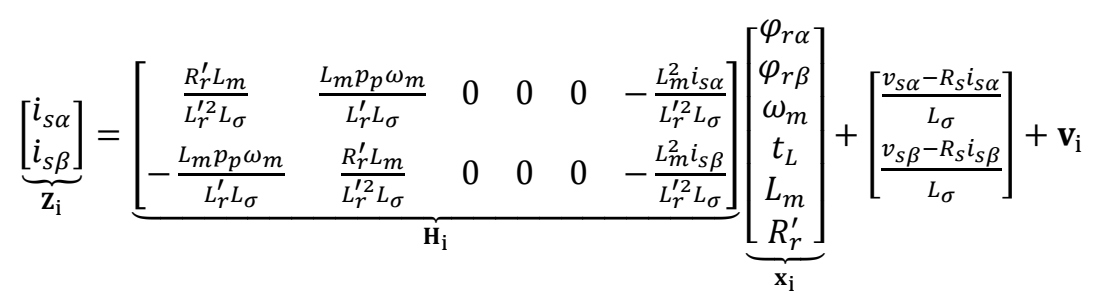

(5)-(8)'de belirtilen ayrıntılı durum uzay gösteriminde; $p_{p}$, kutup çifti sayısı, $v_{s \alpha}$ ve $v_{s \beta}$, stator geriliminin $\alpha \beta$ bileşenleri, $L_{s}$ ve $L_{r}^{\prime}$ sırasıyla, stator ve rotor endüktansı, $L_{\sigma}=\sigma L_{s}$, stator geçici endüktansı, $\sigma=1-\frac{L_{m}^{2}}{L_{s} L_{r}^{\prime}}$, kaçak ya da kuplaj faktörüdür. Ayrıca, $L_{s}=L_{m}+L_{l s}$ ve $L_{r}^{\prime}=L_{m}+L_{l r}$ olarak elde edilmekte olup, $L_{l s}$ ve $L_{l r}$ sirasıly, stator ve rotor kaçak endüktansıdır.

\section{3. İDGKF TASARIMI}

Bu çalışma kapsamında, tam dereceli GKF'nin içerdiği hesap yükünü azaltmak ve ASM'nin hız-algılayıcısız KKD-tabanlı DVK'sının parametre değişimlerine karşı özellikle alan zayıflama bölgesinde gürbüzlüğünü artırmak amacı ile yeni bir İDGKF yöntemi önerilmiştir. Önerilen İDGKF yöntemi ile ASM'nin ölçülmeyen $\varphi_{r \alpha}, \varphi_{r \beta}, \omega_{m}, t_{L}, L_{m}$ ve $R_{r}^{\prime}$ durum ve parametrelerinin eş-zamanlı kestirimi gerçekleştirilmiştir. IDGKF yöntemine ait eşitlikler ve güncelleme adımları [47]'de belirtildiği gibi aşağıda verilmiştir:

- Doğrusal olmayan giriş ve çıkış fonksiyonlarının kestirilen durum ve parametreler etrafinda Taylor Serisi açılımı ile doğrusallaştırılması (Doğrusallaştırma adımı)

$$
\begin{gathered}
\mathbf{F}_{k+1 \mid k}=\left.\frac{\partial \mathbf{f}_{\mathrm{i}}\left(\mathbf{x}_{\mathrm{i}}, \mathbf{u}_{\mathrm{i}}\right)}{\partial \mathbf{x}_{\mathrm{i}}}\right|_{\mathbf{x}_{\mathrm{i}}=\hat{\mathbf{x}}_{\mathrm{i}_{k}}, \mathbf{u}_{\mathrm{i}}=\mathbf{u}_{\mathrm{i}_{k+1}}} \\
\mathbf{H}_{k+1 \mid k}=\left.\frac{\partial \mathbf{h}_{\mathbf{i}}\left(\mathbf{x}_{\mathrm{i}}, \mathbf{u}_{\mathrm{i}}\right)}{\partial \mathbf{x}_{\mathrm{i}}}\right|_{\mathbf{x}_{\mathrm{i}}=\hat{\mathbf{x}}_{\mathrm{i}_{k}}, \mathbf{u}_{\mathrm{i}}=\mathbf{u}_{\mathbf{i}_{k+1}}}
\end{gathered}
$$

- Öngörülen durumların doğrusal olmayan fonksiyonunun ve diş değer hatası kovaryans matrisinin güncellenmesi (Zaman güncellemesi adımı)

$$
\begin{aligned}
& \hat{\mathbf{x}}_{\mathrm{i}_{k+1}}^{-}=\mathbf{f}_{\mathrm{i}}\left(\hat{\mathbf{x}}_{\mathrm{i}_{k}}, \mathbf{u}_{\mathrm{i}_{k+1}}\right) \\
& \mathbf{P}_{k+1}^{-}=\mathbf{F}_{k+1} \mathbf{P}_{k} \mathbf{F}_{k+1}^{T}+\mathbf{Q}
\end{aligned}
$$

- Kalman kazancının, kestirilen durumların ve kestirim hatası kovaryans matrisinin güncellenmesi (Ölçüm güncellemesi adımı)

$$
\begin{aligned}
& \mathbf{K}_{k+1}=\mathbf{P}_{k+1}^{-} \mathbf{H}_{k+1}^{T}\left[\mathbf{H}_{k+1} \mathbf{P}_{k+1}^{-} \mathbf{H}_{k+1}^{T}+\mathbf{R}\right]^{-1} \\
& \hat{\mathbf{x}}_{\mathrm{i}_{k+1}}=\hat{\mathbf{x}}_{\mathrm{i}_{k+1}}^{-}+\mathbf{K}_{k+1}\left(\mathbf{Z}_{k+1}-\mathbf{H}_{k+1} \hat{\mathbf{x}}_{\mathrm{i}_{k+1}}^{-}\right) \\
& \mathbf{P}_{k+1}=\mathbf{P}_{k+1}^{-}-\mathbf{K}_{k+1} \mathbf{H}_{k+1} \mathbf{P}_{k+1}^{-}
\end{aligned}
$$


Burada, F, durum ve girişlerin doğrusal fonksiyonudur. $\mathbf{H}$, ölçüm eşitliğinin doğrusal fonksiyonudur. $\mathbf{P}$, durum ve parametre kestirim hatasının kovaryans matrisidir. $\mathbf{K}$, Kalman kazancıdır. Z, ölçüm vektörüdür. $\hat{\mathbf{x}}_{\mathrm{i}}$, kestirilen durum ve parametrelerin vektörüdür. $\mathbf{Q}$, sistem gürültüsünün kovaryans matrisidir. $\mathbf{R}$, sistem gürültüsünün kovaryans matrisidir.

\section{ASM'NIN HIZ-ALGILAYICISIZ KKD-TABANLI DVK'SI}

Şekil 1'de, bu çalışmada önerilen yeni İDGKF yönteminin parametre kestirimi için kullanıldı̆̆ KKD-tabanlı DVK yöntemi sunulmuştur. Burada geleneksel DVK yönteminde alan ve hız-moment denetçisi olarak kullanılan PI ve ya PID denetçiler yerine [3]'te önerilen alan ve konum KKD'leri $\left(\mathrm{KKD}_{1}\right.$ ve $\left.\mathrm{KKD}_{2}\right)$ kullanılarak ASM'nin hız-algılayıcısız KKD-tabanlı DVK yöntemi oluşturulmuştur. $\mathrm{KKD}_{1}$ ve $\mathrm{KKD}_{2}$ yapıları için [3]'te önerilen $\sigma_{d}$ ve $\sigma_{q}$ kayan manifolt terimleri aşağıdaki gibi tanımlanmıştır:

$$
\begin{aligned}
& \sigma_{d}=\left|\vec{\varphi}_{r}\right|^{r e f}-\varphi_{r d} \\
& \sigma_{q}=\left(\dot{\theta}_{m}^{r e f}-\dot{\theta}_{m}\right)+c\left(\theta_{m}^{r e f}-\theta_{m}\right)=e_{q}+c e_{q}(c>0)
\end{aligned}
$$

$\mathrm{KKD}_{1}$ ve $\mathrm{KKD}_{2}$ 'nin Lyapunov terosine göre kararlılığı için aşağıda belirtilen pozitif fonksiyon seçilmiştir [3, 20]:

$$
V=\frac{1}{2} \sigma_{d}^{2}+\frac{1}{2} \sigma_{q}^{2} \rightarrow \dot{V}=\sigma_{d} \dot{\sigma}_{d}+\sigma_{q} \dot{\sigma}_{q}
$$

$\dot{V}$ fonksiyonunun mutlak negatif tanımlı olması ve böylelikle $\sigma_{d}=0$ ve $\sigma_{q}=0$ alınarak yüzeylerin kesişim noktasında kayan kip durumunun oluşturulması için aşağıdaki eşitlikler tanımlanmıştır:

$$
\begin{aligned}
& \sigma_{d} \dot{\sigma}_{d}=-k_{d}\left|\sigma_{d}\right|^{1 / n_{d}}\left|\sigma_{d}\right| \rightarrow \dot{\sigma}_{d}=-k_{d}\left|\sigma_{d}\right|^{1 / n_{d}} \operatorname{sgn}\left(\sigma_{d}\right) \\
& \sigma_{q} \dot{\sigma}_{q}=-k_{q}\left|\sigma_{q}\right|^{1 / n_{q}}\left|\sigma_{q}\right| \rightarrow \dot{\sigma}_{q}=-k_{q}\left|\sigma_{q}\right|^{1 / n_{q}} \operatorname{sgn}\left(\sigma_{q}\right)
\end{aligned}
$$

burada, $k_{d}>0, k_{q}>0,0<n_{d}<1$ ve $0<n_{q}<1$ olarak alınmaktadır.

$\mathrm{KKD}_{1}$ ve $\mathrm{KKD}_{2}$ 'ye ait kontrol kuramları ya da yapıları, [50]'de belirtildiği gibi, [51]'de tanımlanan anma gücüne ulaşma kanunu ve [52]'de belirtilen ortalama-eşdeğer kontrol kanunun ortak kullanımı ile [3]'de sunulan aşağıdaki eşitlikler ile elde edilmiştir:

$$
\begin{aligned}
& \sigma_{d}=\left|\vec{\varphi}_{r}\right|^{r e f}-\varphi_{r d} \\
& \sigma_{q}=\left(\dot{\theta}_{m}^{r e f}-\dot{\theta}_{m}\right)+c\left(\theta_{m}^{r e f}-\theta_{m}\right)=\left(\omega_{m}^{r e f}-\omega_{m}\right)+c\left(\theta_{m}^{r e f}-\theta_{m}\right)=\dot{e}_{q}+c e_{q}(c=30) \\
& i_{s d}(k+1)=i_{s d}(k) \frac{\sigma_{d}(k+1)-\sigma_{d}(k)}{\frac{R_{r}^{\prime}}{L_{r}^{\prime}} L_{m} T}+\frac{k_{d}\left|\sigma_{d}(k+1)\right|^{1 / n_{d}} \operatorname{sgn}\left(\sigma_{d}(k+1)\right)}{\frac{R_{r}^{\prime}}{L_{r}^{\prime}} L_{m}}\left(k_{d}=0.00015, n_{d}=2\right) \\
& i_{s q}(k+1)=i_{s q}(k) \frac{\sigma_{q}(k+1)-\sigma_{q}(k)}{\frac{3 p_{p L m}}{2 J_{L} L_{r}^{\prime}} T\left|\vec{\varphi}_{r}\right|^{r e f}}+\frac{k_{q}\left|\sigma_{q}(k+1)\right|^{1 / n_{q}} \operatorname{sgn}\left(\sigma_{q}(k+1)\right)}{\frac{3 p_{p} L_{m}\left|\vec{\varphi}_{r}\right|^{r e f}}{2 J_{L} L_{r}^{\prime}}}\left(k_{q}=0.0001, n_{q}=2\right)
\end{aligned}
$$

Şekil 1'de verilen ASM'nin İDGKF'li hız-algılayıcısı KKD-tabanlı DVK'sında akı referansı $\left(\left|\vec{\varphi}_{r}\right|^{\text {ref }}\right)$ değeri, Eşitlik (24)'de belirtildiği gibi tayin edilmiş olup, ASM modeline uygulanan $L_{m}^{r e f}$ değeri ise, ASM'nin anma hızında ve anma hızının altındaki hız değerlerinde çalışması durumunda $L_{m n}$ olarak, anma hızının üzerindeki alan zayıflama bölgesinde çalışması durumunda ise ASM'nin gerçek-zamanlı olarak belirlenen mıknatıslama eğrisi kullanılarak belirlenmiştir. Gerçek-zamanlı ASM deney düzeneği ile yapılan testler sonucu elde edilen mıknatıslama eğrisinin veri adım aralığının daraltılıp sürekli bir fonksiyon olarak tanımlanabilmesi için Matlab “Curve Fitting Toolbox" kullanılarak eğri uydurma yöntemi gerçekleştirilmiştir. 


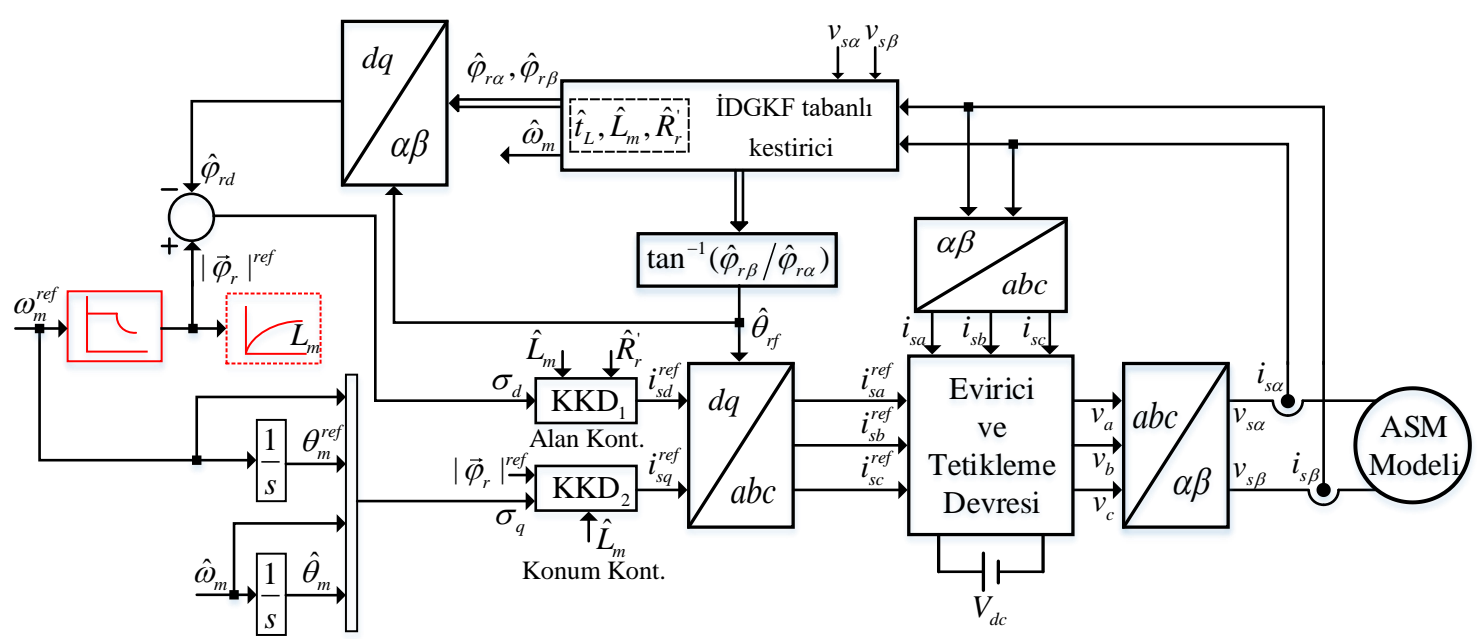

Şekil 1. Yeni İDGKF yönteminin parametre kestirimi için kullanıldı̆̆ı KKD-tabanlı DVK yöntemi

$$
\left|\vec{\varphi}_{r}\right|^{\text {ref }}= \begin{cases}\frac{n_{b}}{n_{m}^{\text {ref }}}\left|\varphi_{r}\right|_{\text {rated }}^{\text {ref }}, & n_{m}^{\text {ref }}>n_{b} \\ \left|\varphi_{r}\right|_{\text {rated }}^{\text {ref }}, & n_{m}^{\text {ref }} \leq n_{b}\end{cases}
$$

Burada $L_{m n}, L_{m}$ 'nin anma değeridir. $\left|\varphi_{r}\right|_{\text {rated }}^{r e f}$, rotor akısı referansının anma değeridir. $n_{m}^{\text {ref }}$, referans rotor mekanik hızıdır. $n_{b}$, ASM'nin mekanik hızının anma değeridir. $\left|\varphi_{r}\right|_{\text {rated }}^{\text {ref }}$ değeri aşağıdaki gibi belirlenmiştir.

$$
\left|\varphi_{r}\right|_{\text {rated }}^{\text {ref }}=\frac{U_{b}}{\omega_{b}}=\frac{\sqrt{2} x \frac{380}{\sqrt{3}}}{2 \pi x 50}=0.987
$$

\section{BENZETIMM SONUÇLARI}

İlk olarak bu çalışmada sunulan ve $\varphi_{r \alpha}, \varphi_{r \beta}, \omega_{m}, t_{L}, L_{m}$ ve $R_{r}^{\prime \prime}$ 'nin eş-zamanlı kestirimi için tasarlanan İDGKF yöntemi, ASM'nin KKD-tabanlı DVK sistemi ile birlikte kullanılarak kestirim başarımı benzetim ortamında test edilmiştir. Önerilen kestirim yönteminin yüksek kestirim başarımına sahip olması sonucunda, KKD-tabanlı DVK'ya, sıfır hızdan anma hızının üzerindeki alan zayıflama bölgesini de kapsayan geniş bir hız aralığında ASM'nin hız-algılayıcısız kontrolünü sağlayabilme özelliği kazandırılmıştır. Benzetim ortamında gerçekleştirilen İDGKF kestiricili ASM'nin hız-algılayıcısız KKD-tabanlı DVK'sında kullanılan ASM modeline ait motor parametreleri Tablo 1'de verilmiştir.

\begin{tabular}{|c|c|c|c|c|}
\hline$P[k W]$ & $f[\mathrm{~Hz}]$ & $J_{T}\left[k g . m^{2}\right]$ & $\boldsymbol{\beta}_{T}[\mathrm{Nm} /(\mathrm{rad} / \mathrm{sn})]$ & $\boldsymbol{P}_{p}$ \\
\hline 2.2 & 50 & 0.055 & 0 & 3 \\
\hline$V[V]$ & $I[A]$ & $\boldsymbol{R}_{s}[\boldsymbol{\Omega}]$ & $\boldsymbol{R}_{r}^{\prime}[\boldsymbol{\Omega}]$ & $L_{l s}[H]$ \\
\hline 380 & 5.5 & 3.03 & 2.53 & 0.0116 \\
\hline$L_{l r}{ }^{\prime}[H]$ & $\boldsymbol{L}_{m}[\boldsymbol{H}]$ & $n_{m}[\mathrm{dev} / \mathrm{dk}]$ & $t_{L}[N . m]$ & \\
\hline 0.0174 & 0.135 & 1000 & 20 & \\
\hline
\end{tabular}

Tablo 1: ASM anma değerleri [43]

IDGKF'ye ait $\mathbf{P}$ kovaryans matrisinin başlangıç değeri ve $\mathbf{Q}$ ve $\mathbf{R}$ kovaryans matrislerinin değerleri deneme yanılma yöntemi ile belirlenmiştir. $\mathbf{P}, \mathbf{Q}$ ve $\mathbf{R}$ köşegen kovaryans matrislerinin belirlenen değerleri aşağıdaki gibi verilmiştir:

$$
\begin{gathered}
\mathbf{Q}=\operatorname{diag}\left\{\begin{array}{cccccc}
1 e-10 & 1 e-10 & 1 e-4 & 1 e-2 & 1 e-7 & 1 e-3\} \\
\mathbf{R}=\operatorname{diag}\{1 e-4 & 1 e-4\}
\end{array}\right. \\
\mathbf{P}=\operatorname{diag}\left\{\begin{array}{llllll}
10 & 10 & 10 & 10 & 10 & 10
\end{array}\right.
\end{gathered}
$$



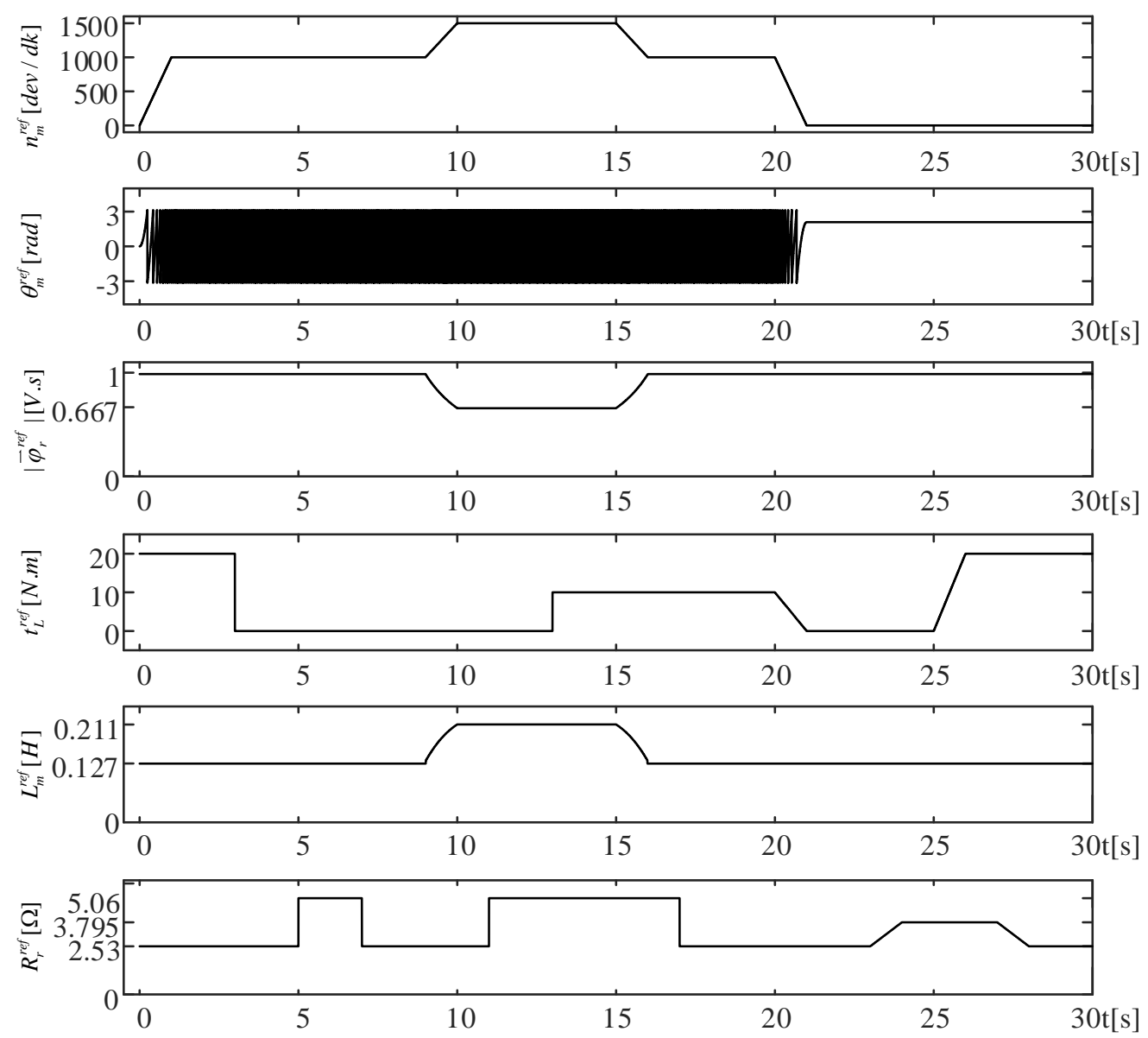

Şekil 2. Önerilen İDGKF ‘nin kestirim başarımı testi için ASM’ye ve KKD-tabanlı DVK'sına uygulanan referans durum ve parametre değişimleri.

İlk kez bu çalışmada önerilen İDGKF'nin kestirim başarımı ve ASM'nin İDGKF'li hız-algılayıcısız KKDtabanlı DVK'sının kontrol başarımı Şekil 2'de verilen referans durum ve parametre değişimleri altında test edilmiştir. Şekil 2'de sunulan çalışma senaryosunun içerdiği zorlayıcı referans durum ve parametre değiş̧imleri aşağıda verilmiştir:

- Tüm hız bölgelerinde kontrol ve kestirim başarımının test edilmesi amacı ile KKD-tabanlı DVK'ya uygulanan referans hız $\left(n_{m}^{r e f}\right)$ değişimi, sıfır hızdan anma hızına kadar ve anma hızının üzerindeki alan zayıflama bölgesini de kapsayan geniş bir hız aralığında değiştirilmiştir. Böylelikle, ASM sıfır hız ve alan zayıflama bölgesini kapsayan geniş bir hız aralığında ileri yönde çalıştırılmıştır.

- $\quad$ ASM modeline uygulanan $t_{L}^{r e f}$ değeri, $t_{L}^{r e f}=0[\mathrm{Nm}]$ ile $t_{L}^{r e f}=20[\mathrm{Nm}]$ aralığında basamak şeklinde ya da doğrusal bir şekilde artırılıp azaltılarak ASM'nin farklı yük kademelerinde ve yüksüz olarak çalışması sağlanmıştır.

- ASM modeline uygulanan $R_{r}^{r e f}$ değeri anma hızında ve alan zayıflama bölgesinde basamak şeklinde $2 x R_{r n}^{\prime}$ değerine yükseltilerek tekrar $R_{r n}^{\prime}$ değerine düşürülmüştür. Sıfır hızda çalışma durumunda ise $R_{r}^{r e f}$ değeri, doğrusal bir şekilde $1.5 x R_{r n}^{\prime}$ değerine yükseltilmiş olup tekrar doğrusal şekilde $R_{r n}^{\prime}$ değerine düşürülmüştür. Burada $R_{r n}^{\prime}, R_{r}^{\prime}$ 'nin anma değeridir.

- KKD-tabanlı DVK'ya uygulanan $\left|\vec{\varphi}_{r}\right|^{r e f}$ değeri, ASM'nin anma hızında ve anma hızının altındaki hız değerlerinde çalışması durumunda Eşitlik (25) ile elde edilen değer olarak belirlenmiş olup, alan zayıflama bölgesine çıkıldığında ise Eşitlik (24)'de de belirtildiği gibi ASM'nin hızı ile ters orantılı olarak azaltılmıştır.

- $\quad L_{m}^{r e f}$ değeri, ASM'nin anma hızını ve anma hızının altındaki hız bölgesini kapsayan ve akı referansının değişmediği durumda $L_{m n}$ olarak belirlenerek ASM modeline uygulanmaktadır. Alan zayıflama bölgesinde ise $L_{m}^{r e f}$ değeri, ASM'nin gerçek-zamanlı deneysel testlerinden elde edilen mıknatıslama eğrisi kullanılarak belirlenmiştir. 

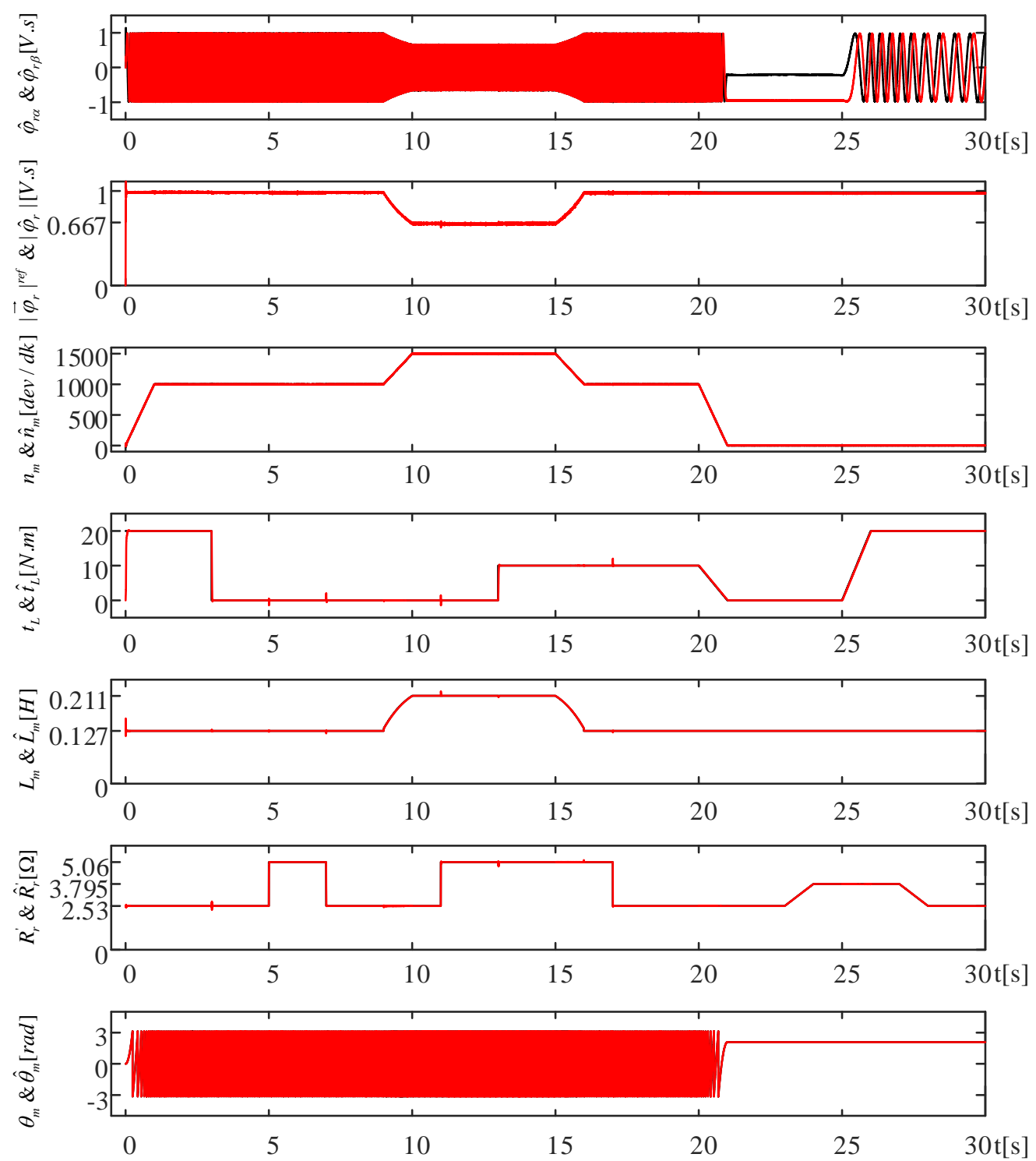

Şekil 3. ASM'nin hız-algılayıcısız KKD-tabanlı DVK'sına ve İDGKF'ye ait benzetim sonuçları.

Şekil 2'de verilen zorlayıcı durum ve parametre değişimlerine karşı önerilen İDGKF yönteminin yüksek kestirim başarımına sahip olduğu Şekil 3 ve 4'te sunulan benzetim ortamındaki kestirim sonuçlarından ve kestirim hatalarından anlaşılmaktadır. $t_{L}^{r e f}$, de meydana gelen basamak şeklindeki ve doğrusal değişimlerin İDKF ile yüksek bir başarımla kestirildiği ve bu değişimler altında $\hat{t}_{L}$ kestirim sonuçlarına bakıldığında herhangi bir hatanın oluşmadığı, yalnızca $t_{L}^{r e f}$,in basamak şeklindeki değişimlerinde $\left|\hat{\varphi}_{r}\right|, \hat{n}_{m}, \hat{R}_{r}^{\prime}$ ve $\hat{L}_{m}$ kestirimlerinde anlık küçük sıçramaların oluştuğu fakat bu sıçramaların hemen sönümlendiği Şekil 3 ve 4 'te görülmektedir. $R_{r}^{r e f}$,de meydana gelen basamak şeklindeki ve doğrusal değişimlere karşı $\hat{R}_{r}^{\prime}$ kestiriminin İDGKF ile yüksek bir performansla sağlandığı ve yalnızca $R_{r}^{r e f}$, in basamak şeklindeki değişimlerinde $\left|\hat{\varphi}_{r}\right|, \hat{n}_{m}$, $\hat{t}_{L}$ ve $\widehat{L}_{m}$ kestirimlerinde anlık küçük sıçramaların oluştuğu fakat bu kestirimlerin gerçek değerlerine çok hızlı bir şekilde yakınsadığı Şekil 3 ve 4'te görülmektedir. ASM'nin alan zayıflama bölgesinde çalışmasının sağlanması için $n_{m}^{r e f}$, in $n_{b}$ değerinin üzerine doğrusal bir şekilde çıkarıldı ̆̆ ve buna bağlı olarak $\left|\vec{\varphi}_{r}\right|^{r e f}$ ve $L_{m}^{r e f}$, in doğrusal olmayan bir şekilde değiştiği Şekil 2 ve 3 'te görülmektedir. Şekil 3 ve 4 'te $\left|\hat{\varphi}_{r}\right|, \hat{n}_{m}, \hat{t}_{L}, \hat{R}_{r}^{\prime}$ ve $\hat{L}_{m}$ kestirimlerine ilişkin sunulan sonuçlar ve kestirim hataları incelendiğinde kestirim sonuçlarının gerçek değerlerine çok hızlı bir şekilde yakınsadığı yalnızca geçici-hal durumunda $\hat{R}_{r}^{\prime}$ kestiriminde önemsenmeyecek derecede bir hatanın oluştuğu ve sürekli-hal durumuna ulaşıldığında bu hatanın ortadan kalktığı görülmektedir. Alan zayıflama bölgesi dışında sıfır hız koşulunda da doğrusal şekilde meydana gelen $t_{L}^{r e f}$ ve $R_{r}^{r e f}$ değişimlerinin ve IDGKF ile 
yüksek bir başarımla kestirildiği ve $\hat{\varphi}_{r} \mid, \hat{n}_{m}$ ve $\hat{L}_{m}$ kestirimlerinde herhangi bir hatanın oluşmadığı Şekil 3 ve 4'te görülmektedir. Bu çalışma ile önerilen yeni IDGKF ile [42]'deki çalışmaya kıyasla ölçülen $i_{s \alpha}$ ve $i_{s \beta}$ durumları kestirilmeden $\hat{t}_{L}$ kestirimi ile birlikte $\left|\hat{\varphi}_{r}\right|, \hat{n}_{m}, \hat{R}_{r}^{\prime}$ ve $\hat{L}_{m}$ kestirimleri de gerçekleştirilerek, ASM'nin hız-algılayıcısız KKD-tabanlı DVK'sının alan zayıflama bölgesinde de kontrol başarımına sahip olması sağlanmıştır.
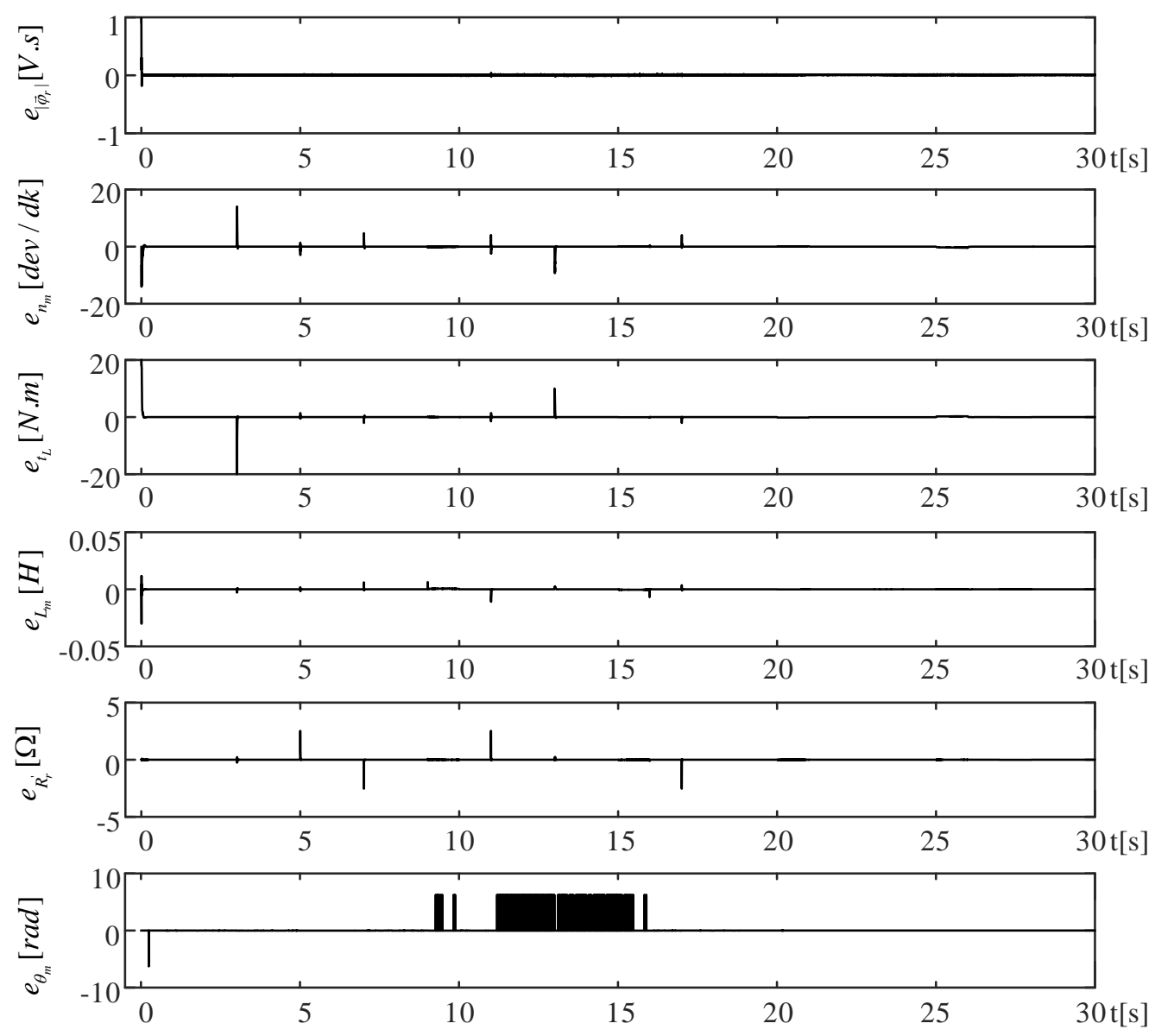

Şekil 4. Önerilen İDGKF'ye ait kestirim hataları.

\section{SONUÇ VE YORUMLAR}

Bu çalışmada, ASM'lerin geniş bir hız aralığında (sıfır hız ile anma hızının üzerindeki alan zayıflama bölgesini de kapsayan) hız-algılayıcısız dinamik kontrolü için yeni bir İDGKF algoritması önerilmiştir. Önerilen İDGKF yönteminin kestirim başarımı ise ASM'nin KKD-tabanlı DVK'sı üzerinde Matlab/Simulink benzetim ortamında test edilmiştir. ASM'nin sıfır hız durumunda ve anma hızının üzerindeki alan zayıflama bölgesinde sargı sıcaklığı ve çalışma frekansından dolayı $R_{r}^{\prime}$ 'de, yük koşullarından dolayı $t_{L}$ 'de ve sargı gerilim sınırından dolayı $\left|\vec{\varphi}_{r}\right|^{r e f}$ ve $L_{m}$ 'de meydana gelebilecek değişimler İDKF ile yüksek bir başarımla kestirilmiştir.

Benzetim ortamında İDKF algoritması ile birlikte oluşturulan ASM'nin hız-algılayıcısız KKD-tabanlı DVK'lı sürücü sisteminden elde edilen sonuçlar önerilen yeni İDGKF kestiricisinin ve KKD-tabanlı DVK ASM sürücü sisteminin geniş bir hız aralığında yüksek kestirim ve kontrol başarımına sahip olduğunu göstermektedir. İlk kez bu çalışmada önerilen yeni İDKK tabanlı kestiricinin, ölçülen $i_{s \alpha}$ ve $i_{s \beta}$ durumları kestirilmeden [42] ve [47]'den farklı olarak $\hat{t}_{L}$ kestirimi ile birlikte $\left|\hat{\varphi}_{r}\right|, \hat{n}_{m}, \hat{R}_{r}^{\prime}$ ve $\hat{L}_{m}$ kestirimleri de gerçekleştirilerek, ASM'nin hızalgılayıcısız KKD-tabanlı DVK'sının alan zayıflama bölgesinde de kontrol başarımına sahip olması sağlanmıştır. 


\section{KAYNAKLAR}

[1] HOLMES, D.G., MC GRATH, B.P., PARKER, S.G., "Current Regulation Strategies for Vector-Controlled Induction Motor Drives”, IEEE Transactions on Industrial Electronics, 59 (10), 3680-3689, 2012.

[2] ASTROM, K.J., HAGGLUND, T., "The future of PID control”, Control Engineering Practice, 9 (11), 11631175, 2001.

[3] BARUT, M., BOGOSYAN, S., "Sensorless Sliding Mode Position Control of Induction Motors Using Braided Extended Kalman Filters", IEEE International Symposium on Industrial Electronics, 2268-2273, Vigo, Spain, 2007.

[4] BARAMBONES, O., ALKORTA, P., "Position Control of the Induction Motor Using an Adaptive SlidingMode Controller and Observers", IEEE Transactions on Industrial Electronics, 61 (12), 6556-6565, 2014.

[5] MORINO, R., PERESEDA, S., VALIGI, P., "Adaptive input-output linearizing control of induction motors", IEEE Transactions on Automatic Control, 38 (2), 208-221, 1993.

[6] GUZINSKI, J., ABU-RUB, H., "Speed Sensorless Induction Motor Drive with Predictive Current Controller", IEEE Transactions on Industrial Electronics, 60 (2), 699-709, 2013.

[7] AlKortA, P., BARAMBONES, O., CORTAJARENA, J.A, ZUBIZARRRETA, A., "Efficient Multivariable Generalized Predictive Control for Sensorless Induction Motor Drives", IEEE Transactions on Industrial Electronics, 61 (9), 5126-5134, 2014.

[8] TEJA, A.V.R., CHAKRABORTY, C., MAITI, S., HORI, Y., “A New Model Reference Adaptive Controller for Four Quadrant Vector Controlled Induction Motor Drives", IEEE Transactions on Industrial Electronics, 60 (2), 3757-3767, 2012.

[9] ORLOWSKA-KOWALSKA, T., DYBKOWSKI, M., SZABAT, K., “Adaptive Sliding-Mode Neuro-Fuzzy Control of the Two-Mass Induction Motor Drive Without Mechanical Sensors", IEEE Transactions on Industrial Electronics, 57 (2), 553-564, 2010.

[10] TALLA, J., LEU, V.Q., ŠMÍDL, V., PEROUTKA, Z., “Adaptive Speed Control of Induction Motor Drive with Inaccurate Model", IEEE Transactions on Industrial Electronics, 65 (11), 8532-8542, 2018.

[11] SIRA-RAMÍREZ, H., GONZÁLEZ-MONTAÑEZ, F., CORTÉS-ROMERO, J.A., LUVIANO-JUÁREZ, A., "A Robust Linear Field-Oriented Voltage Control for the Induction Motor: Experimental Results", IEEE Transactions on Industrial Electronics, 60 (8), 3025-3033, 2013.

[12] SUETAKE, M., DA SILVA I.N., GOEDTEL, A., "Embedded DSP-Based Compact Fuzzy System and Its Application for Induction-Motor V/f Speed Control", IEEE Transactions on Industrial Electronics, 58 (3), 750-760, 2011.

[13] GUO, L., PARSA. L., "Model Reference Adaptive Control of Five-Phase IPM Motors Based on Neural Network, IEEE Transactions on Industrial Electronics, 59 (3), 1500-1508, 2012.

[14] GADOUE, S.M., GIAOURIS, D., FINCH, J.W., "Sensorless Control of Induction Motor Drives at Very Low and Zero Speeds Using Neural Network Flux Observers", IEEE Transactions on Industrial Electronics, 56 (8), 3029-3039, 2009.

[15] SHIAU, L., LIN, J., "Stability of sliding-mode current control for high performance induction motor position drives", IEEE Proceedings on Electric Power Applications, 148 (1), 69-75, 2001.

[16] KAYNAK, O., ERBATUR, K., ERTUGRUL, M., "The fusion of computationally intelligent methodologies and sliding-mode control-a survey”, IEEE Transactions on Industrial Electronics, 48 (1), 417,2001

[17] HUNG, J.Y., GAO, W., HUNG, J.C., "Variable structure control: a survey", IEEE Transactions on Industrial Electronics, 40 (1), 2-22, 1993.

[18] UTKIN, V.I., "Sliding mode control design principles and applications to electric drives", IEEE Transactions on Industrial Electronics, 40 (1), 23-36, 1993.

[19] YAN Z., JIN, C., UTKIN, VI., "Sensorless sliding-mode control of induction motors”, IEEE Transactions on Industrial Electronics, 47(6), 1286-1297, 2000.

[20] RODIC, M., JEZERNIC, K., "Speed-sensorless sliding-mode torque control of an induction motor", IEEE Transactions on Industrial Electronics, 49 (1), 87-95, 2002.

[21] ARAB MARKADEH, G.R., SOLTANI, J., "Sliding-mode control for speed sensorless induction machine drive using an adaptive nonlinear rotor flux observer", COMPEL The International Journal for Computation and Mathematics in Electrical and Electronic Engineering, 25 (4), 940-963, 2006.

[22] BENNASSAR, A., ABBOU, A., AKHERRAZ, M, ESSALMI, A., BARARA, M., MAHMOUDI, H., "Sensorless Sliding Mode Control of Induction Motor Based on Luenberger Observer Using Fuzzy Logic Adaptation Mechanism", Journal of Theoretical and Applied Information Technology, 65 (1), 130-136, 2005.

[23] DI GENNARO S., DOMÍNGUEZ J.R., MEZA, M.A., "Sensorless High Order Sliding Mode Control of Induction Motors with Core Loss", IEEE Transactions on Industrial Electronics, 61 (6), 2678-2689, 2014. 
[24] JING, C., CHAONAN, T., "Sliding mode repetitive equivalent control for induction motor based on hardware-in-loop system", $43^{\text {rd }}$ Annual Conference of the IEEE Industrial Electronics (IECON 2017), 31163121, Beijing, China, 2017.

[25] REZGUI, S.E., BENALLA, H., "New Robust and Mechanical Sensorless Scheme for SVM Inverter Fed Induction Motor Drive Using Variable Structure Controllers and MRAS", Arabian Journal for Science and Engineering, 38 (6), 1449-1458, 2013.

[26] TRAORÉ, D., PLESTAN, F., GLUMINEAU, A., DE LEON J., "Sensorless Induction Motor: HighOrder Sliding-Mode Controller and Adaptive Interconnected Observer", IEEE Transactions on Industrial Electronics, 55 (11), 3818-3827, 2008.

[27] INAN, R., BARUT, M., "Bi input-extended Kalman filter-based speed-sensorless control of an induction machine capable of working in the filed-weakening region", Turkish Journal of Electrical Engineering \& Computer Sciences, 22 (3), 588-604, 2014.

[28] HUANG, M.S., LIAW, C.M., "Improved field-weakening control for IFO induction motor", IEEE Transactions on Aerospace and Electronic Systems, 39(2), 647-659, 2003.

[29] SHIN, M.H., HYUN, D.S., "Speed sensorless stator flux-oriented control of induction machine in the field weakening region", IEEE Transactions on Power Electronics, 18 (2), 580-586, 2003.

[30] MATSUSE, K., KUBOTA, H., "Deadbeat flux level control of high power saturated induction servo motor using rotor flux observer”, IEEE Industrial Application Society Annual Meeting, 409-414, Dearborn MI, USA, 1991.

[31] KLAES, N.R., "Parameter identification of an induction machine with regard to dependencies on saturation”, IEEE Transactions on Industry Applications, 29 (6), 1135-1140, 1993.

[32] ZHAO, L., HUANG, J., CHEN, J., YE, M., "A Parallel Speed and Rotor Time Constant Identification Scheme for Indirect Field Oriented Induction Motor Drives", IEEE Transactions on Power Electronics, 31 (9), 6494-6503, 2016.

[33] CAO, P., ZHANG, X., YANG, S., “A Unified-Model-Based Analysis of MRAS for Online Rotor Time Constant Estimation in an Induction Motor Drive", IEEE Transactions on Industrial Electronics, 64 (6), 4361-4371, 2017.

[34] WANG, B., ZHAO, Y., YU, Y., WANG, G., XU, D., DONG, Z., “Speed-Sensorless Induction Machine Control in the Field-Weakening Region Using Discrete Speed-Adaptive Full-Order Observer", IEEE Transactions on Power Electronics, 31 (8), 5759-5773, 2016.

[35] ZHANG, Y., BAI, Y., YANG, H., ZHANG, B., "Low Switching Frequency Model Predictive Control of Three-Level Inverter-Fed IM Drives with Speed Sensorless and Field-Weakening Operation", IEEE Transactions on Industrial Electronics, Early Access, 1-11, 2018.

[36] HABIBULLAH, M., LU, D.D., “A Speed-Sensorless FS-PTC of Induction Motors Using Extended Kalman Filters”, IEEE Transactions on Industrial Electronics, 62 (11), 6765-6778, 2015.

[37] HABIBULLAH, M., LU, D.D., XIAO, D., FLETCHER, J.E., RAHMAN, M.F., "Predictive Torque Control of Induction Motor Sensorless Drive Fed by a 3L-NPC Inverter", IEEE Transactions on Industrial Informatics, 13 (1), 60-70, 2017.

[38] DENGHAN-AZAD, E., GAdOUE, S., ATKInSON, D., SLATER, H., BARRASS, P. BLAABJERG, F., "Sensorless Control of IM for Limp-Home Mode EV Applications", IEEE Transactions on Power Electronics, 32 (9), 7140-7150, 2017.

[39] DENGHAN-AZAD, E., GADOUE, S., ATKInSON, D., SLATER, H., BARRASS, P. BLAABJERG, F., "Sensorless Control of IM Based on Stator-Voltage MRAS for Limp-Home EV Applications", IEEE Transactions on Power Electronics, 33 (3), 1911-1921, 2018.

[40] ZAKY, M.S., KHATER, M.M., SHOKRALLA, S.S., YASIN, H.A., "Wide-Speed-Range Estimation with Online Parameter Identification Schemes of Sensorless Induction Motor Drives", IEEE Transactions on Industrial Electronics, 56 (5), 1699-1707, 2009.

[41] ORLOWSKA-KOWALSKA, T., TARCHALA, G., DYBKOWSKI, M., "Sliding-mode direct torque control and sliding-mode observer with a magnetizing reactance estimator for the field-weakening of the induction motor drive", Mathematics and Computers in Simulation, 98, 31-45, 2014.

[42] INAN, R., BARUT, M., ZERDALI, E., DEMIR, R., YILDIZ, R., "FPGA Realization of EKF Based SpeedSensorless Drive Working in Field-Weakening Region", International Conference on Engineering Technology and Innovation (ICETI 2017), 81-88, Sarajevo, Bosna-Hersek, 2017.

[43] INAN, R., DEMIR, R., BARUT, M., "Hybrid Estimator Based Direct Vector Control of IM with SpeedSensor", OHU Journal of Science, 7 (2), 612-623, 2018.

[44] ALONGE, F., CIRRINCIONE, M., PUCCI, M., SFERLAZZA, A., “A Nonlinear Observer for Rotor Flux Estimation of Induction Motor Considering the Estimated Magnetization Characteristic", IEEE Transactions on Industry Applications, 53 (6), 5952-5965, 2017. 
[45] LIU, L., DU, X., SHEN, S., "Indirect field-oriented torque control of induction motor considering magnetic saturation effect: error analysis", IET Electric Power Applications, 11 (6), 1105-1113, 2017.

[46] RUAN, J., WANG, S., "Magnetizing Curve Estimation of Induction Motors in Single-Phase Magnetization Mode Considering Differential Inductance Effect", IEEE Transactions on Power Electronics, 31 (1), 497506, 2016.

[47] DEMIR, R., BARUT, M., YILDIZ, R., "Asenkron Motorların Hız-Algılayıcılı Doğrudan Vektör Kontrolü için İndirgenmiş Dereceli Genişletilmiş Kalman Fitresi Tabanlı Parametre Kestirimi", Otomatik Kontrol Ulusal Toplantısı (TOK 2017), 562-567, İstanbul, Türkiye, 2017.

[48] YIN, Z., ZHAO, C., LIU, J., ZHONG, Y., "Research on Anti-Error Performance of Speed and Flux Estimator for Induction Motor Using Robust Reduced-Order EKF", IEEE Transactions on Industrial Informatics, 9 (2), 1037-1046, 2013.

[49] ALSOFYANI, I.M., IDRIS, N.R.N., "Simple Flux Regulation for Improving State Estimation at Very Low and Zero Speed of a Speed Sensorless Direct Torque Control of an Induction Motor", IEEE Transactions on Power Electronics, 31 (4), 3027-3035, 2016.

[50] ZERDALI, E., BARUT, M., "Novel version of bi input-extended Kalman filter for speed-sensorless control of induction motors with estimations of rotor and stator resistances, load torque, and inertia", Turkish Journal of Electrical Engineering \& Computer Sciences, 24, 4525-4544, 2016.

[51] HUNG, J.Y., GAO, W., HUNG, J.C., "Variable structure control: a survey", IEEE Transactions on Industrial Electronics, 40 (1), 2-22, 1993.

[52] SAHIN, C., SABANOVIC, A. GOKASAN, M., "Robust position control based on chattering free sliding modes for induction motors", IEEE 1995 International Conference on Industrial Electronics, Control, and Instrumentation, 512-517, Orlando, FL, New York, ABD, 1995. 